\title{
SYSTEM SOFTWARE FRAMEWORK FOR SYSTEM OF SYSTEMS AVIONICS
}

\author{
Roscoe C. Ferguson, Benjamin L. Peterson, Hiram C. Thompson \\ United Space Alliance, LLC \\ Houston, Texas
}

\begin{abstract}
Project Constellation implements NASA's vision for space exploration to expand human presence in our solar system. The engineering focus of this project is developing a system of systems architecture. This architecture allows for the incremental development of the overall program. Systems can be built and connected in a "Lego style" manner to generate configurations supporting various mission objectives.
\end{abstract}

The development of the avionics or control systems of such a massive project will result in concurrent engineering. Also, each system will have software and the need to communicate with other (possibly heterogeneous) systems. Fortunately, this design problem has already been solved during the creation and evolution of systems such as the Internet and the Department of Defense's successful effort to standardize distributed simulation (now IEEE 1516). The solution relies on the use of a standard layered software framework and a communication protocol.

A standard framework and communication protocol is suggested for the development and maintenance of Project Constellation systems. The ARINC 653 standard is a great start for such a common software framework. This paper proposes a common system software framework that uses the Real Time Publish/Subscribe protocol for framework-to-framework communication to extend ARINC 653.

It is highly recommended that such a framework be established before development. This is important for the success of concurrent engineering. The framework provides an infrastructure for general system services and is designed for flexibility to support a spiral development effort.

\section{Introduction}

NASA's plan to deploy a system of systems architecture to implement project Constellation requires the development of an adaptable and networked system. This architecture will contain hardware and software in every system of the collective. A wise plan of action would be first to develop a common framework to allow for successful system integration and maintenance. This technique has been used to develop successful ventures such as the Internet and the Department of Defense's (DOD) distributed simulation infrastructure. The Internet has a set of standards including RFC 791 [1] and the DOD's distributed simulation standard that has evolved to IEEE 1516 [2]. In each case, such an infrastructure has allowed for the integration and maintenance of a system of distributed heterogeneous platforms.

This successful model can be used to provide the same benefits for NASA's future space systems. The creation of a standardized system software framework is recommended in support of this effort. ARINC 653 provides an infrastructure for safety-critical software and serves as an excellent starting point. This paper proposes a framework that can be used to extend ARINC 653 to create the adaptable and networked systems required for the system of systems architecture.

\section{ARINC 653 Systems}

ARINC 653 is a software standard interface for avionics applications. The standard is intended to provide a platform for safety-critical systems. Central to the standard are the concepts of Space and Time Partitioning. Space Partitioning provides isolation for applications executing on the same computing platform. Applications are encapsulated in containers defined as partitions. Time Partitioning isolates the CPU time allocated to partitions using a time-slotted scheduler [3]. 
ARINC 653 defines an Application/Executive (APEX) software interface between applications software and the Operating System [4]. The Application Programming Interface (API) provides support for partition management, process management, time management, inter-partition communication, intra-partition communication, and health monitoring [5]. COTS Real Time Operating System (RTOS) products are now providing ARINC 653 solutions. Most implementations use the memory management unit (MMU) of the target processor to implement Space Partitioning. Each partition contains its own application and OS. A central OS (partition manager) manages the collective of partitions and controls Time Partitioning (Figure 1).

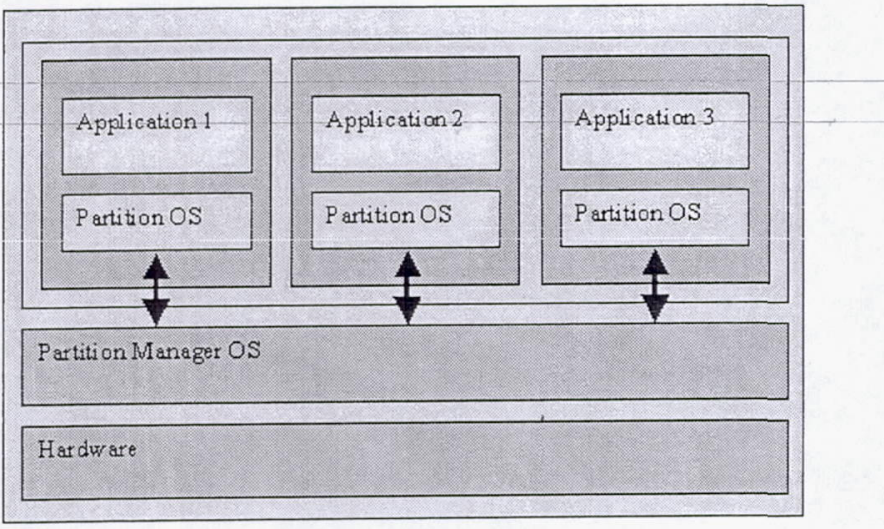

Figure 1. ARINC 653 Architecture

\section{System Software}

System Software provides the infrastructure and services for applications in real time systems. It is composed of an OS and a software layer providing system control and application services. Each system in the NASA system of systems architecture will have a computer-processing platform that will require system software. It is likely that individual systems in the collective will be developed by various vendors. However, the system must fit together and work in a seamless manner. The creation of a standard system software framework can be instrumental in the success of NASA's new engineering task. Since system software provides the infrastructure for applications, NASA can provide a template to enforce a standard to ensure successful system integration. The template could be used to speed the development of new systems and provide a familiar architecture for understanding and maintaining existing systems. The template can be implemented as middleware to be distributed and deployed for future development efforts. This is similar in concept to the Run-Time Infrastructure (RTI) that is used to implement IEEE 1516 standard for distributed simulation systems. The RTI is the middleware required to implement the High Level Architecture (HLA) [2].

ARINC 653 compatible RTOSs provide an excellent starting point to achieve the goal of standardized system software. First, the design is tailored towards the creation of safety-critical systems. Second, it provides a standard API to support system development. However, a RTOS is only a part of system software. The suggested framework concept can be completed by the creation of an additional layer that makes use of the standard API and other RTOS support. For framework-to-framework communication, a standard protocol is required for data exchange. A successful and popular protocol used in real time distributed systems is Real Time Publish/Subscribe. In 2004, this protocol became an official standard [6] and has been used successfully in existing military platforms [7]. It is suggested that this protocol be used to support the distributed network required for the system of systems architecture.

\section{System Software Framework}

Every variation of computer software can be reduced to a common processing template. Inputs are acquired, processed, and the results made available for output. Typically, system software provides the mechanisms for input, output, and system flow control. Applications perform the task of processing and transforming the inputs into outputs. Despite the fact all software reduces to a common template, the "wheel is reinvented" for most new development efforts. For system software, flow control is implemented using various techniques that perform the same overall operation. Various forms of support operations are also reimplemented. In addition, the designs of these systems are not adaptable. The support for inputs 
and outputs are intertwined with the processing logic.

The design for the framework is based on the simplification of a system into inputs, processing, and outputs. A prime focus is to decouple inputs and outputs from processing using a database interface. Also, a common set of system services are integrated and packaged into a central entity. The framework therefore, consists of the four main components. These are Input Modules, Logic Modules, Output Modules, and a Central Manager.

Separate input, logic, and output modules provide three main benefits. First, input and output are the two processing elements that are most likely to be affected by modeling and simulation. Therefore, this design will allow for logic to be written and isolated from these components. Models can be interchanged for hardware and vice versa with no impact to the core logic. Second, the logic of a system can be developed in the absence of real hardware. Third, multiple logic modules can be associated with the same input module, and multiple output modules can be associated with the same logic module. This allows flexibility and support for code reuse. This could all be made possible via the use of a database interface. This interface provides a data abstraction layer common to all modules. Input Modules can acquire data from various sources or devices, but must store the results into a database. Logic Modules can use the database to access data acquired by Input Modules and store results into the database. Output Modules can use the database to access data produced by Logic Modules.

To increase modularity and portability, the Input, Logic, and Output Modules should use a standard interface method. A concept is to replace most module-to-module API with the Central Manager. Logic Modules may need to work together to perform activities. This can be implemented via a single interface command (similar to the OS SVC Supervisor Call command) to make the request for services. The request is routed to the Central Manager to dispatch the service. This can eliminate the problems with module specific API function interfaces. For example, to develop and compile module specific software, there are interface dependencies between developers. Developer A must wait on Developer B to provide a function interface and if this interface is constantly changing (function protocol, function name), confusion and delay can occur. However, an SVC like call that accepts a service name and generic input, output, and status information can be managed from a central entity. Each module must register available services with the Central Manager. If an SVC request is made for an unregistered service, the requesting module can take action, or the Central Manager can allow system operation only where all dependencies have been registered. There will always be development dependencies, but they can be minimized. Services provided by the Central Manager can use an API format since this interface is to be standardized.

The Central Manager is the core that provides all services required to support the framework. It can be considered to be the system software. From an architectural point of view (Figure 2), the Logic Modules implement the "domain" logic for a system. The Input Modules, Output Modules, and Central Manager provide support to realize the "domain" logic. The Input Modules provide support for data acquisition, the Output Modules provide support for operations such as commanding and displays, and the Central Manager provides the system software.

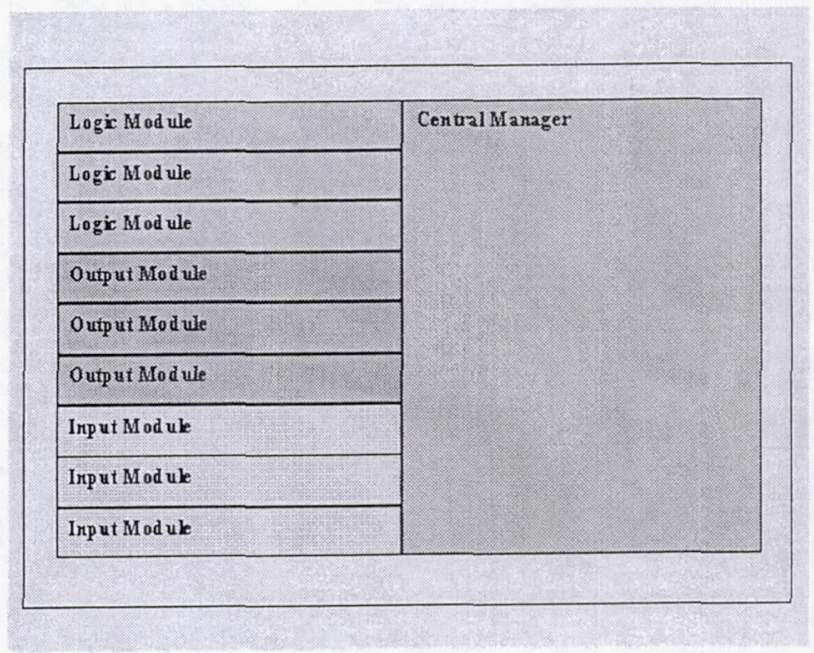

Figure 2. SSW Framework Architecture

\section{Central Manager}

The Central Manager uses the API and services provided by an ARINC 653 compatible 
RTOS to implement a standard system software layer. This layer provides services to and controls the Input, Logic, and Output Modules of the framework. It is composed of a Registration Manager, Time and Event Sequence Manager, Service Manager, Health Manager, Configuration Manager, Initialization/Patch Manager, Telemetry/Logging Manager, External System and Heartbeat Manager, Database Manager, Data Publishing Manager, and Data Subscription Manager (Figure 3).

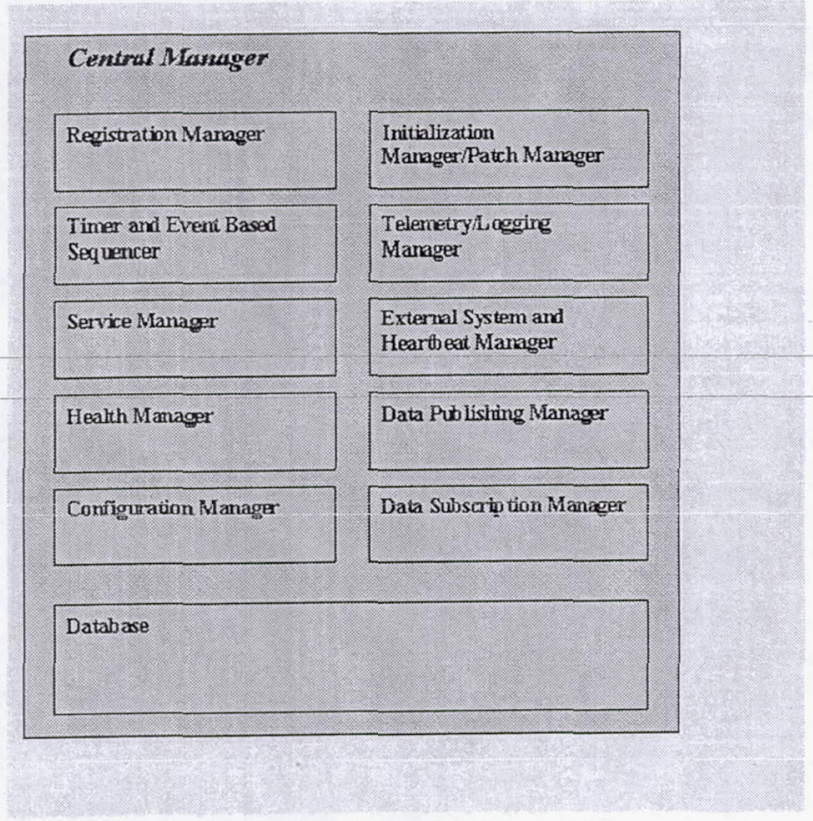

Figure 3. Framework Central Manager

The Registration Manager provides a registration interface between modules and the Central Manager. Modules can register services to be used by other modules and can register for services provided by other modules. Modules can also register for read/write access to parameters in the database. A registration interface provides a runtime method to provide and acquire services. This allows modules to be added and removed from the system without a total recompile. This benefit is only available if the supporting RTOS provides the capability for dynamic loading and linking of multiple object files.

The Timer and Event Sequencer provide the service required to invoke modules based on either a timer or events. It implements the flow control for the system. The sequencer provides a hybrid infrastructure supporting an executive, priority based task manager, and state machines. The sequencer is implemented using RTOS services such as Process Control and Event Management. It provides the concept of major and minor cycles to structure cyclic operations. Input, Logic, and Output Modules are associated with one or more sequencer control methods.

The Service Manager provides the single SVC interface in support of module-based services. Modules register the intent to provide services using the Registration Manager. The Service Manager invokes the module service when requested via the interface.

The Health Manager provides a centralized system error handler. The system could provide an API to be called by each module to report error conditions. The manager would consist of a table mapping error conditions to policy functions. The policy function performs actions in response to errors. This could work with the Configuration Manager to determine how to configure or safe the system in response to failures and reconfigure after recovery. It could also be implemented using fault trees in support of the analysis of error paths. ARINC 653 provides an interface and services for health management. This manager could use the ARINC interface to implement this service. A benefit is that the framework version can be integrated to work with the other framework modules to provide advanced capability.

The Configuration Manager configures the system based on system state. It contains tables that map the relationships for modules and data sources required for proper operation. It interfaces with the Registration Manager, Health Manager, and the External System and Heartbeat Manager to analyze system state for modules. It also interfaces with the Timer and Event Sequencer to activate or deactivate the operation of modules. For example, if Module A depends on Module B and data from an external module for proper operation and Module B has not been registered, then Module A would not be allowed to execute. Also, if Module A is operational, but data from the external module is no longer available, then Module A will be shutdown until the data is operational in the future. 
The Initialization/Patch Manager provides an interface to allow for module initialization. The intent is to load an object file and invoke an initialization function for each Input, Logic, and Output Module. The concept of a separate object file for each module provides support for flexible and dynamic systems. New capability can be incorporated without a system recompile and existing modules can be temporarily replaced to support simulation. The initialization function for each module can include all logic required to initialize that module including the use of the Registration Manager API to register services and other required items. The manager also provides support for patch capability. The simplest approach is to replace an existing object file. The change will take effect at the next system initialization event. This manager relies on the linking and loading capability of the RTOS.

The Telemetry and Logging Manager provides services required to report and record system status. For Telemetry, this system can gather data from the database for transmission to the ground or other destinations. The system could also store information to logs for post mortem analysis. An interface API allows the modules to make request for the logging of information.

The External System/Heartbeat Manager communicates with external systems to determine system of systems configuration. It listens for the heartbeats of external system and sends the heartbeat for its system. It provides information to the Configuration Manager for the determination of system operability in relation to external systems.

The Database provides an API and a repository to store and request most data used by the system. It consists of a collection of named parameters and buffers. The buffers would be used to store block data from Input Modules. The Database could be populated from either internal modules or data from external modules. Also, each parameter has an associated status to indicate data availability. The Database provides support for centralized operations such as data scaling, limit/range checking, and data conversion. The Database could work with the Sequencer to support the activation of modules based on the arrival of data from external frameworks.
The Data Publishing Manager provides the service to send data to remote systems collected from the database. The Data Subscription Manager provides the service to receive data from remote systems to store into the database. Each uses the Real Time Publish/Subscribe protocol.

\section{Example Design Implementation}

This section provides a concept of execution using the framework concept. It first provides the configuration of a hypothetical system and then follows with scenarios through various lifecycles and system operation.

\subsection{Configuration}

The platform of the demonstration configuration consists of three physical transportation modules and an outpost (Figure 4). The first module is a transport module that serves as a crew habitat. This module is the same for all mission configurations. However, it can be attached to one of two propulsion modules. There is a short duration and a long duration propulsion module. Depending on the mission, the crew module can be attached to either the long duration or short duration propulsion module (Figure 5). Once in space, the transportation configuration can dock with one of many outposts (Figure 6).

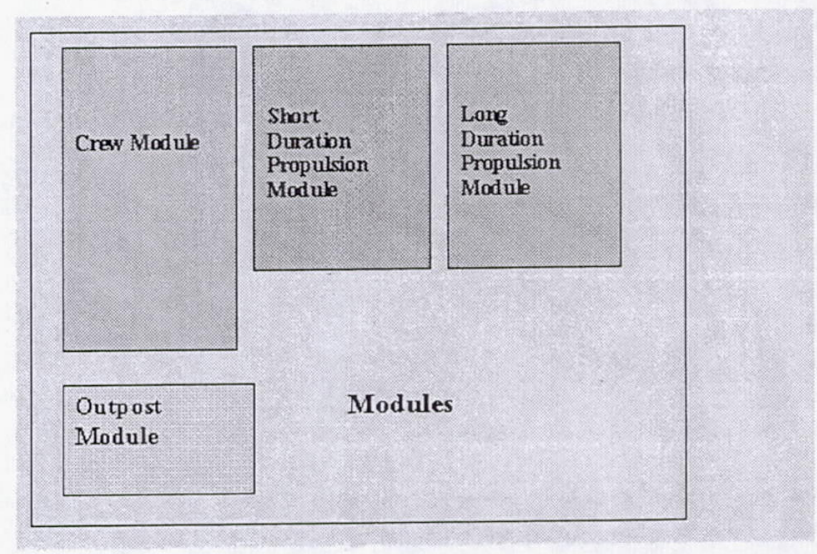

Figure 4. System of Systems Modules

The modules are networked using Ethernet technology. The designers of the system have implemented a multiple Ethernet bus infrastructure that provides a network path for commanding and a network path for general data distribution (for displays, less critical operations, etc.). This 
decreases the traffic load on the command bus, thus increasing determinism. Each node of the network is a PowerPC Single Board Computer (SBC) with dual Ethernet transceivers to support the multiple bus architecture. It can be either connected to one or both networks (Figure 7).

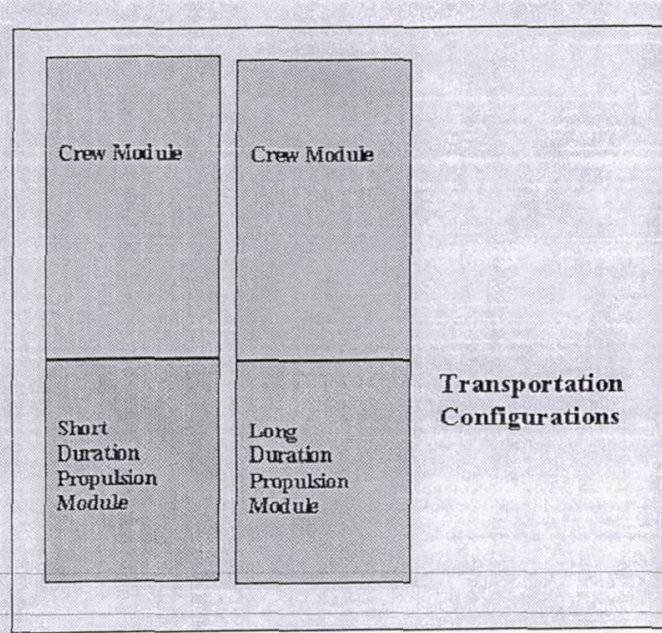

Figure 5. Transportation Configurations

The Crew Module contains a Guidance Navigation and Control (GNC) SBC, a Systems Management (SM) SBC, a Crew Interface SBC, and a Situational Awareness SBC. For simplicity, assume that the GNC, SM, and Crew Interface $\mathrm{SBCs}$ are interfaced to both networks and the Situational Awareness SBC is interfaced to the Data Distribution Network. Both the Propulsion Modules consists of Engine Control SBCs that are interfaced to both networks. The Outpost Module consists of Environment Control and Crew Interface SBCs. The Environment Control SBCs and the Crew Interface SBCs are connected to both networks.

For simplicity, this configuration does not address redundancy. Its purpose is to support the operational scenarios of the framework concept.

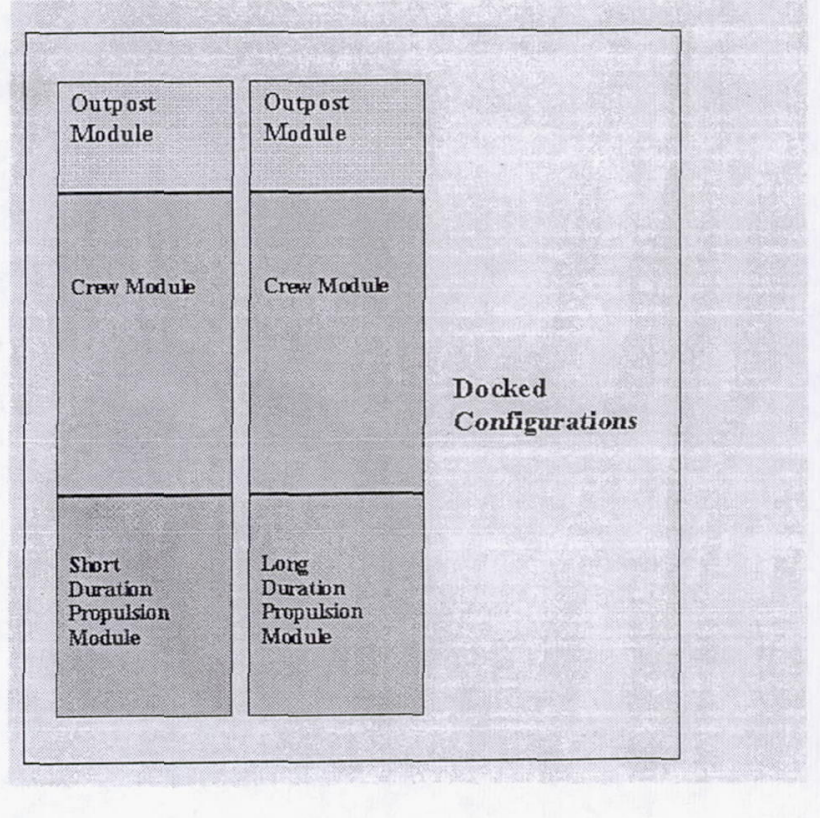

Figure 6. Docked Configurations

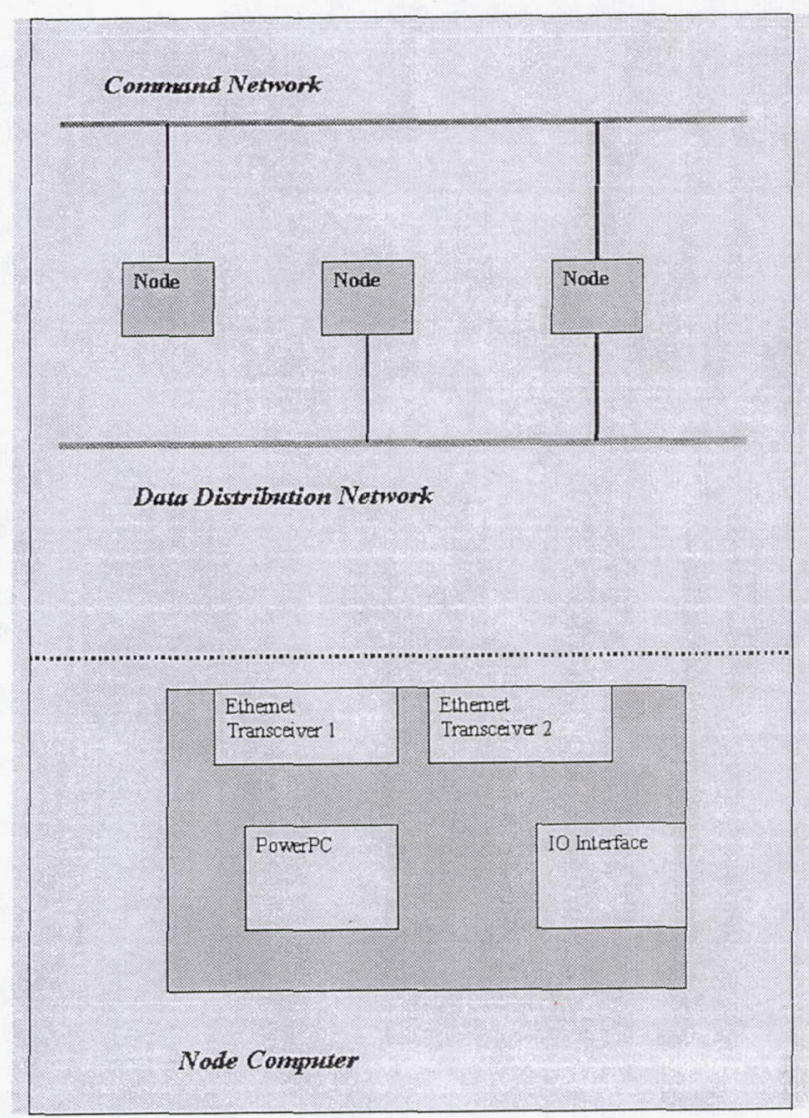

Figure 7. System Network 


\subsection{Node Interface Technology}

For this sample implementation, the framework is implemented using VxWorks ARINC 653 for OS support, NDDS for network support, and a PowerPC hardware platform (Figure 8).

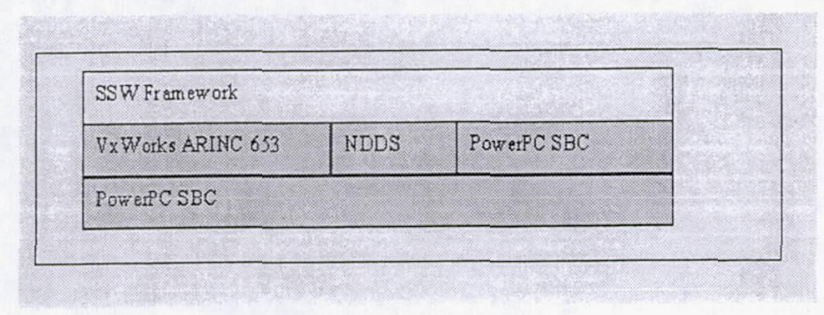

Figure 8. System Implementation

\subsection{Crew Module Computer Requirements}

The GNC computer reads hardware instruments to determine the location and orientation of the vehicle and sends commands to the Propulsion Modules for control.

The GNC computer can receive commands from the Crew Interface Module to adjust the behavior of the output commands.

The GNC computer uses independent algorithms for the control of the two Propulsion Modules.

In the Docked Configuration, the GNC Computer enters a safe mode to prevent activity with the Propulsion Modules.

In the Docked Configuration, the GNC Computer is connected to a simulator where the crew can practice maneuvers using the actual spacecraft interface.

The GPC computer will enter a safe mode if it fails to communicate the Propulsion Modules.

The SM computer receives commands from the Crew Interface computers of the Crew Module and Outpost Module.

The SM computer sends display data over the Data Distribution Network.

The Crew Interface computer sends crew commands over the Command Network.
The Situational Awareness computer receives data over the Data Distribution Network and displays information to the crew.

\subsection{System Software Real-Time Infrastructure (RTI)}

The first step of the project would be to create a Real-Time Infrastructure using the system software framework. Assume the RTI was created using the $\mathrm{C}$ language. The software implementation was designed into two layers (Figure 9). The first layer implements all interfaces of the framework. The second layer provides an interface between the $\mathrm{OS}$ and the first layer using a concept similar to those of RTOS Board Support Packages (BSP). The BSP uses a table driven interface to configure the services of the Central Manager.

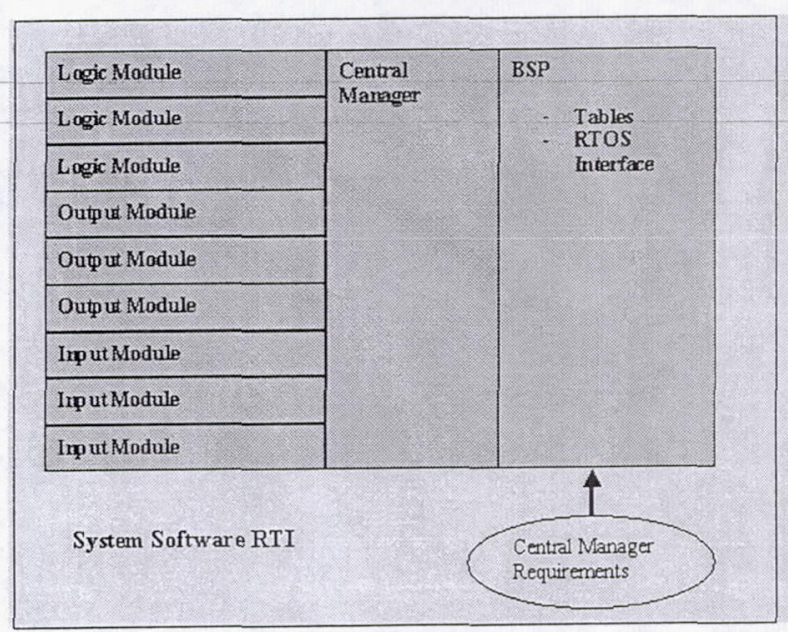

Figure 9. SSW RTI

\subsection{Crew Module Software Design}

To implement the requirements for the Crew Module GNC computer, framework modules were implemented and the Central Manager tables of the framework BSP were populated with configuration data.

The GNC framework modules consist of a Sensor Input Module, a Short Duration Propulsion Crew Command Input Module, a Long Duration Propulsion Crew Command Input Module, a Short Duration Propulsion Logic Module, a Long Duration Propulsion Logic Module, a Short Duration Propulsion Command Output Module, a Short Duration Propulsion Display Data Output 
Module, a Long Duration Propulsion Command Output Module, a Long Duration Propulsion Display Data Output Module, a Maneuver Input Crew Command Simulation Module, and a Maneuver Simulation Output Module.

The Initialization Manager is configured to the object files for the current configuration and invokes the initialization function for each module.

The External System and Heartbeat Manager have been configured to listen for heartbeats from both Propulsion Modules, and the Outpost Module.

The Configuration Manager has been configured for the following configuration trees:

- Good Short Duration Propulsion Heartbeat, No Internal Failure, No Outpost Heartbeat, Sensor Input Module registered, a Short Duration Propulsion Crew Command Input Module registered, Short Duration Propulsion Logic Module registered, Short Duration Propulsion Command Output Module registered, Short Duration Propulsion Display Data Output Module registered. If all true, then place GNC computer in active mode, else place GNC computer in safe mode.

- Good Long Duration Propulsion Heartbeat, No Internal Failure, No Outpost Heartbeat, Sensor Input Module registered, a Long Duration Propulsion Crew Command Input Module registered, Long Duration Propulsion Logic Module registered, Long Duration Propulsion Command Output Module registered, Long Duration Propulsion Display Data Output Module registered. If all true, then place GNC computer in active mode, else place GNC computer in safe mode.

- Good Outpost Heartbeat, Maneuver Input Crew Command Simulation Module registered, Long Duration Propulsion Logic Module registered, Maneuver Simulation Output Module registered, Long Duration Propulsion Display Data Output Module registered. If all true, then place GNC computer in simulation mode, else place GNC computer in safe mode.
The Database has been configured to support parameters for display output data, input buffers collected from the Sensor Input Module, and parameters that represent commands from the Crew Interface computer of the Crew Module. The Database also stores parameters for commands to be sent to the Propulsion Modules.

The Data Publisher Manager has been configured to gather display output parameter data from the Database to publish over the Data Distribution Network.

The Data Subscription Manager has been configured to gather Propulsion Output parameter data from the Data Distribution Network and populate the Database.

The Timer/Event Sequencer has been configured to execute the Sensor Input Module, Propulsion Logic Modules, and Command Output Modules at $25 \mathrm{~Hz}$ if operational based on the Configuration Manager. Also, the Display Output Modules have been configured to execute at $2 \mathrm{~Hz}$. The Command Input Module has been configured to execute on the arrival of commands from the Crew Interface computer.

The software for the SM computer, Crew Interface computer, and the Situational Awareness computer has been implemented using the RTI configured to support their operations.

\subsection{Concept Of Execution From GNC Computer Point of View}

The Crew Module contains a mode switch to indicate the three possible configurations (Short Duration Transportation Configuration, Long Duration Transportation Configuration, or Docked Configuration). The Central Manager loads a different set of object modules based on the mode switch as a level of protection vs. loading all software and deactivating modules.

For the Long and Short Duration Transportation Configuration Modes, the Configuration Manager places the GNC computer in either active or safe mode. For control in the active mode, the Timer/Event Sequencer invokes the Sensor Input Module, Propulsion Logic Modules, and Command Output Modules at $25 \mathrm{~Hz}$. These three modules are invoked in order by the 
executive. The Sensor Input Module acquires data from the hardware and populates the database with parameters. The Propulsion Logic Module reads the database, performs processing data and produces output to the database. The Command Output Module reads the command from the database and sends it to the Propulsion Module. The Output Module checks the database status of the command to ensure that it is valid and not stale. The Propulsion Display Data Output Module is executed by the Timer/Event Sequencer executive at $2 \mathrm{~Hz}$. It acquires data from the database and prepares it to be published over the Data Distribution Network by the Data Publisher Manager. The Situational Awareness computer receives the published data and formats it for crew displays. The Timer/Event Sequencer invokes the Crew Command Input Module when the Data Subscription Manager receives a published command from the Crew Interface computer.

For the Docked Configuration Mode, the Configuration Manager places the GNC computer into the safe mode when the Outpost Heartbeat is detected. The crew can set the mode switch to load the GNC computer with the Docked Configuration software. Once loaded, the GNC computer is active in a simulation mode where the crew can practice for long duration mission maneuvers. The Maneuver Input Crew Command Simulation Module populates the database with input values based on crew input and models. The actual Long Duration Propulsion Logic Module populates the database with output command. The Maneuver Simulation Output Module processes the commands and provides feedback to the models to close the loop.

\subsection{Discussion}

Several benefits can be abstracted from the use of the system software framework in the example design.

The first benefit is the use of an ARINC 653 compatible RTOS for the OS portion of the framework. The hardware design for the system used separate physical computers in the same module to perform various tasks. However, these applications could have been combined into a single processing platform isolated by ARINC 653 partitions. ARINC 653 platforms provide designers with flexible options to produce safety critical systems. For the design example, applications were implemented in separate computers, but partitions could have been used to isolate critical components of the application inside a single computer.

The example project developed a RTI for the system software framework with a BSP interface. The RTI would save the project from reimplementing system software for every computer in every module. The RTI could be developed and verified once before distribution to other development efforts. Other development projects could implement platform specific system software capabilities by configuring tables in the BSP.

The flexibility of the example project framework interface allowed the spacecraft configuration to support on-board training using the actual spacecraft control and feedback interface. The input and output modules that supported maneuver control were replaced with modules providing simulation. The real maneuver control logic module was used unchanged. This same flexibility could have been used during the development phase where the logic modules could be developed and matured before the availability of flight hardware components. The database interface concept allows for the seamless interchange of hardware and models.

\section{The Configuration Manager and External} System/Heartbeat Manager of the example project framework allowed the computers of each module to recognize the configuration state and adapt as required. The design of the example system had the crew use a mode switch to indicate the system configuration while the framework verified the state. This was performed to prevent the case where a system failure could trick a computer identifying the incorrect state. Also, the loading of mode specific software provided another layer of protection to prevent the execution of inadvertent software. However, the system could also be designed to support automatic configuration. All available software modules could be loaded at initialization. The Configuration Manager and External System/Heartbeat Manager could activate the software modules via control of the Timer/Event Sequencer.

The use of the Real-Time Publish/Subscribe protocol allows for the seamless integration of 
distributed computers on the network. The protocol can be used to distribute both commands and data. The framework can be relieved of the duties of performing low-level network programming. Also, the framework does not have to be aware of how and which nodes are using its published data. New nodes can be added and removed from the network with ease.

\subsection{Framework for "Open Source" RTOS Products}

Concerns of the use of COTS products in safety critical systems include lack of insight into the internal design and the ability to fix problems in a short timeline. For these reasons, programs have considered the use of "open source" RTOS products. An example of such an RTOS is eCos. It is an open source real-time operating system that is royalty-free and intended for embedded applications [8].

The framework can also be incorporated to execute as a layer over this "open source" RTOS. It could be used to implement the flight control computer of an avionics system where the designers would have total insight into the design and the ability to make fixes in a short timeline. The framework could also work in a system that contained both COTS ARINC 653 and "open source" RTOS systems. The "open source" RTOS systems could support control loop software, while the COTS ARINC 653 systems could support critical monitor applications.

\subsection{Conclusion}

System Software provides the infrastructure and services for applications in real time systems. It is composed of an OS and a software layer providing system control and application services. This software is normally re-implemented during various development efforts. This usually results in various software implementations performing common operations.

NASA's plan to deploy a system of systems architecture to implement project Constellation requires the development of an adaptable and networked system. This architecture will contain system software in every system of the collective.
NASA can implement a common system software framework to force an architecture template to support the successful integration and maintenance of this engineering challenge. ARINC 653 compatible RTOS platforms can be extended with a standardized layer to provide a viable solution.

\section{Acknowledgments}

The authors wish to thank Mark Lostracco, Brian Watson and Wendy Wilkinson for their support of this work.

\section{References}

[1] Information Sciences Institute, University of Southern California, September 1981, Internet Protocol DARPA Internet Program Protocol Specification, Marina del Rey, California, Ch.3.

[2] Department of Defense, November 14, 1997, High Level Architecture Run-Time Interface Programmers Guide, Ver. 1.0, Rel. 3, pp. 7-13.

[3] Parkinson, Paul, 2003, Safety Critical Software Development for Integrated Modular Avionics, Alameda, California, Wind River, pp. 2-8.

[4] Child, Jeff, March 2004, Safety Critical Software Choices Expand, COTS Journal Online.

[5] Airlines Electronic Engineering Committee, May 16, 2005, Draft 1 of Project Paper 653:

Avionics Application Software Standard Interface, Part 3 - Conformity Test Specification, Reference 05-125/SWM - 97, Annapolis, Maryland, ARINC, p.1.

[6] Pardo-Castellote, Gerardo, January 2005, OMG Data Distribution Service: Real-Time Publish/Subscribe Becomes a Standard, RTC, pp.41-45.

[7] Murphy, Brett, July 2004, Fabrics and PublishSubscribe Schemes: A Net-Centric Blend, COTS Journal Online.

[8] Sgandurra, Robert, August 2004, An Introduction to eCos, COTS Journal Online.

\section{$24^{\text {th }}$ Digital Avionics Systems Conference}

October 30, 2005 